\title{
LA MOTIVACIÓN DEL USO DE LAS TIC EN LA FORMACIÓN DE PROFESORADO EN EDUCACIÓN AMBIENTAL
}

\author{
The motivational use of ICT \\ in Environmental Education teachers' training
}

Joaquín Paredes ${ }^{1}$ Rogério Dias de Arruda ${ }^{2}$

Resumen: En este artículo se analizan narrativas relacionadas con el papel de la motivación que el uso de las TIC trae a la formación de gestores medioambientales. Se construyeron casos donde para recoger los datos se utilizó entrevistas y aplicación de cuestionarios. Se hizo la interpretación de los datos con técnicas de Análisis de Contenido y la inclusión de una herramienta de mapas conceptuales. Se hizo evidente que las TIC se utilizan de manera general, inespecífica y poco organizada por profesores y estudiantes. Los docentes presentan formas de integración y usos diferentes: expositivos, banco de recursos, colaborativos, teniendo mayor presencia los primeros. Sin embargo, los resultados apuntan a que no hay consenso entre los participantes sobre la motivación que el uso de las TIC aporta a la formación de profesorado en Educación Ambiental y que ello es debido a las narrativas que se generan.

Palabras clave: Motivación. TIC. Tecnologías de la información y la comunicación. Formación de profesorado. Educación ambiental.

\begin{abstract}
This article examines narratives concerning the role of motivation that the use of ICT brings to the training of environmental managers. Cases were built from collected data of interviews and questionnaires. The interpretation of data used content analysis methods and a concept mapping tool. It became clear that ICT is used in general, in a non-specific, and poorly organized way by teachers and students. Teachers present forms of integration and different uses such as exhibition, repository banks, collaborative work, all having greater possible expository uses. However, the results suggest that there is no consensus among participants on the motivation that the use of ICT gives to teacher training in environmental education, and that is due to the narratives that are generated.
\end{abstract}

Keywords: Motivation. ICT. Information and communication technologies. Teacher training. Environmental education.

\footnotetext{
${ }^{1}$ Departamento de Didáctica y Teoría de la Educación, Facultad de Formación de Profesorado y Educación, Universidad Autónoma de Madrid (UAM). Avda. Francisco Tomás y Valiente, 3, 28049, Madrid, España. joaquin.paredes@uam.es

${ }^{2}$ Laboratório de Pesquisa e Estudos em Psicologia Social, Universidade Federal do Rio Grande. Rio Grande, RS, Brasil.
} 


\section{Introducción}

El trabajo que presentamos es parte de un proyecto de investigación cualitativa más amplio que se desarrolló en la Universidad Federal de Río Grande (FURG), Brasil. Tal investigación - que se realizó sobre dos programas de posgrado: el Programa de Doctorado Interuniversitario en Educación Ambiental (DIEA), de España, y el Programa de Posgrado en Educación Ambiental de la FURG (PPGEA), de Brasil- se propuso analizar los usos de las Tecnologías de la información y la comunicación (TIC) en la formación de gestores medioambientales en esos dos programas.

Para obtener éxito en esa integración es necesario que no se haga uso de las TIC de manera indiscriminada o con poco fundamento didáctico (OJEDA, 2008), una vez que los recursos tecnológicos ponen a disposición de los actores del proceso de enseñanza universitaria (profesores y estudiantes) nuevas posibilidades y ventajas (MERMA, 2008; PAREDES; ESTEBANELL, 2005).

El problema principal que orientó la investigación es el siguiente: ¿Cómo contribuyen las TIC a la formación de gestores medioambientales en programas de posgrado?

En este artículo se hace referencia, en particular, a una de sus contribuciones: la comprensión de la motivación que el uso de las TIC trae a la formación de gestores medioambientales.

La temática de la motivación tiene una larga tradición, algo abandonada en el campo de la didáctica y con aproximaciones en el ámbito de la psicología básica interesada en el rendimiento educativo de los estudiantes. Según Mallart (2008), la motivación es una fuerza interna de los estudiantes compuesta por pensamiento, creencias y emociones que surge y pervive en ellos orientada a la realización de tareas propuestas en la docencia de una materia. Esta fuerza está presente tanto en el seguimiento activo de la exposición de información por parte del profesor como en la realización de actividades y proyectos de autoestudio, vale decir en todo el elenco didáctico y organizativo de enseñanza que quepa imaginar. Esto queda dicho de una forma más analítica mediante una de las teorías para explicar las diferencias en la motivación de los alumnos por aprender, la de la orientación a metas relacionadas con el logro (ALONSO-TAPIA, 2007), que define otras tantas orientaciones motivacionales (al aprendizaje, al resultado, a la evitación). A su vez, otros estudios han analizado las relaciones entre la motivación y las expectativas de los estudiantes (ALONSO-TAPIA, 2005). Una temática similar a la motivación es la de la autorregulación (BOEKAERTS; NIEMIVIRTA, 2000).

Esta descripción analítica no acaba de explicar el papel de la motivación, que queda comprometida tanto por los contextos (ALONSO-TAPIA, 2005) como por la singularidad de cada interacción en el aula (ALONSO-TAPIA; HUERTAS; RUIZ, 2010; MALLART, 2008).

La motivación que propicia la introducción de las TIC en la enseñanza es un hito reiterado en la literatura que promueve y caracteriza tal introducción, y está generalmente ligada a la mejora de los procesos comunicativos (por ejemplo CABERO, 2006). Este sesgo de la literatura tiene que ver con que los estudiantes de posgrado analizados suelen ser los que acabarán siendo formadores (como ocurre en el presente estudio), y las TIC se convierten en una "bala de plata" de los docentes que va a resolver múltiples problemas relacionados con el funcionamiento de las clases y los resultados que obtengan los estudiantes. En esta última dirección se analizarán los casos abordados. 
El tema de la interrelación entre TIC y motivación, teniendo en cuenta esta contextualización a la formación de docentes, ha sido estudiado, entre otros, por Cox, Preston y Cox (1999), Hendriks (1999), Karsenti (1999), Karsenti y Lira-Gonzalez (2010, 2011), García et al. (2002), Tejedor y García-Valcárcel (2006), y Tejedor, García-Valcárcel y Prada (2009). Las principales aportaciones de estos estudios indican que hay una serie de factores que pueden contribuir a la motivación para utilizar las TIC. Los principales son:

- las creencias en el valor de las TIC para el aprendizaje y la enseñanza;

- las actitudes de los sujetos hacia las TIC;

- la percepción de competencia para utilizarlas;

- las dificultades de uso;

- los recursos disponibles.

En general, esos autores coinciden que hay una visión optimista sobre la integración y el uso de las TIC en la docencia, en la medida en que consideran como aspectos motivadores, por ejemplo, la posibilidad de tornar las clases más interesantes, mejorando la presentación de materiales didácticos; el aumento del intercambio de conocimientos mediante la reducción de las barreras temporales y espaciales; la autonomía; y la facilidad del acceso a la información. Otros autores, conviene señalar, no son tan optimistas en una visión unidimensional (disponer de infraestructuras como son las TIC) en los procesos de cambio en la enseñanza y que hay nuevos problemas asociados como cambios en la metodología practicada, participación de los estudiantes o formación específica de los docentes, como luego se dirá.

Abordamos un problema complejo como suelen ser todos los educativos. La entrada de las TIC es una nueva variable. Aunque quizá los docentes que las introducen son capaces de apreciar un cambio de la actitud de sus estudiantes en múltiples direcciones, nunca se explicita en qué medida se hace evidente tal motivación, posiblemente porque es un tema de difícil estudio y que sólo recientemente está siendo recuperado por parte de la ciencia de la didáctica.

Se plantea, entonces, que con TIC hay mejor implicación de los estudiantes en aquella didáctica que las incluye. ¿Esto es posible? Es necesario intentar una aproximación densa al campo de estudio para poder responderlo.

\section{Material y métodos}

De la revisión realizada y que se indicaba más arriba, existen estudios de corte cualitativo, entre ellos Karsenti y Lira-González (2010), donde se han incluido las siguientes técnicas: entrevistas individuales, análisis semi-estructurados y Análisis de Contenido. El análisis de resultados se desarrolló de la siguiente forma: los datos recolectados a través de entrevistas pasaron por transcripciones, donde surgieron varios conceptos. La prueba de los conceptos se hizo codificando los datos (con ayuda del software NVivo). Con el intento de ajustar y refinar los conceptos, el proceso de codificación fue verificado reiteradas veces. Al final, los conceptos fueron agrupados en categorías.

Por nuestra parte, se puso en marcha un estudio de naturaleza cualitativa (GOETZ; LECOMPTE, 1988) porque permite realizar: un análisis situado (en escenarios, contextos, espacios determinados) y holístico, en el que el investigador interpreta y da sentido a elementos de la realidad; y un análisis interpretativo (dando significados), en el que el lenguaje es una 
herramienta fundamental para reconstruir, dando vida a lo que se relata. Se trata, por lo tanto, de un enfoque mixto, en que la comparación de datos y la triangulación (entre las opiniones de profesores, estudiantes y la teoría) se constituyen ejes que garantizan la construcción de un conocimiento científicamente válido y confiable (HERNÁNDEZ; FERNÁNDEZ; BAPTISTA, 2006).

Las técnicas elegidas fueron, de un lado, la entrevista semiestructurada (con una adaptación en forma de cuestionario) (MINAYO, 1995) y, por otro, el estudio de caso (DE PABLOS, 1996), técnica ésta que concretamos en los dos programas de educación ambiental estudiados. La entrevista nos permite una reconstrucción densa y triangulada con los sujetos participantes en la misma en cada caso. A su vez, los casos permiten que en la comparación emerjan categorías no apreciadas inicialmente, así como una reformulación de los mismos que los completa.

\section{Procedimientos, técnicas y recogida de datos}

Sobre los procedimientos y técnicas de recolección de datos, la primera acción necesaria fue definir los criterios para que profesores y estudiantes pudiesen participar de la investigación. Por las limitaciones de espacio, la muestra que se trabajará en este artículo hace referencia exclusivamente al estudio con los sujetos del Programa DIEA.

Con relación a los profesores, fueron establecidos los siguientes criterios: a) el profesor debería tener vínculo permanente con el programa o ser colaborador por más de 4 años; y b) el profesor ya debería haber dado al menos un curso en el programa a que estaba vinculado. De un total de 23 profesores, 22 fueron considerados aptos.

En relación a los estudiantes, como único criterio, común a los dos programas, se definió: los estudiantes deberían estar cursando el doctorado en sus respectivos programas. Tal definición apuntó que los estudiantes del DIEA fuesen los que ingresaron en el año académico 2004/05. Había 24 estudiantes que cumplían el requisito.

Se elaboraron instrumentos de recolección de datos, para profesores y estudiantes, constituido cada uno de ellos por 11 cuestiones abiertas, además de un apartado introductorio que contextualizaba la temática y objetivos de la investigación. Ese instrumento fue utilizado como un guión para entrevista y también como cuestionario a ser contestado a distancia, por correo electrónico. En los cuestionarios había una pregunta específica sobre motivación.

Simultáneamente, se procedió a contactar a los responsables de los cursos y exponerles los objetivos de la investigación con objeto de obtener sus colaboraciones y participaciones, concediendo una cita o comprometiéndose a contestar el cuestionario que sería enviado por internet.

Fue necesaria la aplicación de protocolos de entrevista a distancia (a través de correo electrónico) para alcanzar los objetivos de la investigación, teniendo en cuenta que no hubo condiciones logísticas y financieras de contacto presencial con todos los sujetos que participaron de la investigación.

En un primer momento se consiguió realizar cuatro entrevistas presenciales a profesores y 12 aceptaron hacerlo mediante cuestionario para ser contestado a través de internet; dos contestaron negativamente, alegando falta de tiempo o desinterés por la temática; y otros cuatro no contestaron. De los 12 cuestionarios enviados a través del correo electrónico, se 
recibió seis contestados (los otros seis profesores no se manifestaron al respecto), configurando 10 participaciones de 22 posibles.

Pensando inicialmente en aportar el ambiente del curso y mejorar la triangulación, se concordó que también sería importante traer a la investigación las opiniones de otros estudiantes sobre los usos de las TIC en sus formaciones. Ello permitiría complementar el recorrido investigativo, así como mejorar el análisis e interpretación de los datos, teniendo en cuenta el origen académico de los estudiantes que ingresan en un Posgrado de Educación Ambiental, provenientes de las más diversas áreas del conocimiento.

El procedimiento para la recolección de datos con los estudiantes fue el mismo que se había utilizado con los profesores: cuando no fue posible la cita, la obtención de las informaciones se dio a través del correo electrónico. En la página del programa estaban los nombres de los 32 estudiantes en esa promoción. Se envió mensajes a todos ellos, pero se recibió ocho contestaciones automáticas informando que las direcciones electrónicas no existían. De los otros 24 estudiantes, se recibió la contestación de 10 de ellos; nueve, positivamente, en el sentido de poder enviar el cuestionario por internet, y una contestación negativa de una estudiante informando que, a pesar de su nombre estar en la lista, el hecho de no haber conseguido una beca la hizo desistir del curso.

\section{Procedimientos para el análisis de los datos}

En esta sección se enfoca la fase que trata del análisis e interpretación de los datos, basada en el Análisis de Contenido. Este tipo de análisis es un proceso donde los textos originales, en una primera etapa, pasan por la desconstrucción, que consiste en un examen detallado y en la fragmentación del texto en unidades de base, y, a continuación, por su categorización, que establece una nueva relación entre esas unidades, permitiendo el agrupamiento de elementos semejantes, así como condiciones para una mayor profundización en el análisis e interpretación de los datos (BARDIN, 2008; PUGLISI, 2007).

Además de coincidir en los conceptos del Análisis de Contenido, Bardin y Puglisi afirman que para entender las situaciones del día a día es fundamental considerar el ambiente donde ocurren tales situaciones, así como el interior de determinados espacios de interacción personal e institucional, teniendo en cuenta que son mediados por modalidades técnicas de construcción y transmisión de mensajes, cada vez más complejas, en los días actuales.

Los casos son construidos mediante un procedimiento de diálogo constante o permanente con los mismos (BORONAT, 2005). Se valoró la utilización de un programa de análisis cualitativo, pero al ser tan discreta la muestra, se optó por un procedimiento de diálogo permanente con los datos recogidos. Por otra parte, las evidencias de diferentes fuentes que se utilizaron para la construcción de los casos nos ofrecen un estudio de caso denso (STAKE, 2007).

\section{El surgimiento de la categoría general y de las unidades de análisis}

Para el análisis de los casos en relación al problema, la motivación y las TIC, el proceso de construcción de categorías tuvo inicio ya en la elaboración del instrumento de recolección de los datos, pues las preguntas del cuestionario se basaron en aspectos presentes en el problema de la investigación. 
De ese modo, se configuraron las siguientes temáticas: los usos de las TIC, el papel del profesor, la motivación con el uso de las TIC, la brecha digital, la didáctica con las TIC, la postura crítica ante las TIC, el proceso de enseñanza-aprendizaje con las TIC y el futuro del gestor ambiental con las TIC. Esas temáticas no se constituyeron en categorías a priori, una vez que ellas podrían no convertirse en una categoría o una unidad de análisis, ya que eran susceptibles de sufrir cambios y adecuaciones tras la realización de las entrevistas y de la aplicación de los cuestionarios, y principalmente durante y después el análisis de datos.

Después de la transcripción de las entrevistas y de la recepción de los cuestionarios contestados fue realizada la lectura de esos documentos objetivando la categorización de los datos. Tras leer las contestaciones de los participantes, los datos, ahora ya identificados como unidades de registro, fueron nuevamente agrupados por respuestas afines. De esa interpretación emergió una gran categoría general, a saber, "El papel del profesor en la formación de profesorado en Educación Ambiental con las TIC”, y las unidades de análisis que la componen son las siguientes:

- Postura del profesor ante las TIC

- Tipos de usos de las TIC

- Motivación con el uso de las TIC

- Brecha digital

- El estudiante en la formación del profesorado con las TIC y su perspectiva futura

En el apartado siguiente se hará referencia a una de esas unidades de análisis, que es la "Motivación" con el uso de las TIC. Como el resto de apartados, en el análisis fueron emergiendo elementos y relaciones con los que construimos una narración densa de lo ocurrido con las TIC, en forma de malla conceptual, y unas narrativas de los propios sujetos, que iban concordando unas con otras al reconstruir los casos.

\section{Resultados}

\section{Análisis de los usos de las TIC relacionados con la motivación que propician (o no) las TIC a los profesores y estudiantes del Posgrado en Educación Ambiental}

No se puede hablar sobre la motivación que surge entre los estudiantes con el uso de las TIC sin relacionarla con los tipos de usos de los recursos tecnológicos.

Preguntados si utilizan los recursos de las TIC en sus prácticas, con pequeñas reservas, se observa que el uso de las TIC es una práctica común entre profesores y estudiantes. Sin embargo, esa práctica no es uniforme, presentando variaciones en cuanto a las formas de integración y a los usos de las TIC. Asimismo, la referencia a las variaciones de tal práctica engloba las actividades de los participantes en las dos sedes, o sea, en sus universidades de origen (en las nueve universidades que integran el DIEA) y en el Centro Nacional de Educación Ambiental (CENEAM). Se destaca ese aspecto porque, en relación al CENEAM, es prácticamente unánime la opinión de que las condiciones para el uso de las tecnologías no son buenas. 
La mala conexión con la internet del CENEAM puede contribuir para que haya esa variación en cuanto a los tipos de usos de las TIC, pues, recordando a McClintock (2001), una de las etapas del proceso de integración de las herramientas tecnológicas en el trabajo docente requiere una conexión técnicamente mejorada, así como que esa conexión llegue a todos los laboratorios de informática y aulas con ordenadores con calidad uniforme.

Hemos identificado a través de las entrevistas (donde se utilizarán los siguientes códigos: de P1 a P10 para los profesores, y de E1 a E7 para los estudiantes) varios usos según el contexto y el análisis de la realidad educativa que hace un profesor: uso de construcción colaborativa del conocimiento, donde hay mejoras notables en los procesos de comunicación; de mejora de la comunicación fuera del aula; y de utilizar las TIC como banco de recursos. Algún profesor llega a desarrollar proyectos mediante una plataforma de teleformación.

Se expresan distintos niveles de integración y usos (básico, intermedio y avanzado) entre los 10 profesores, pues es posible deducir que hay diferencias de dominio de las TIC entre ellos, una vez que algunos hacen un uso de comunicación básica y otros un uso más integrado a sus prácticas.

Recientemente, algunas investigaciones se preguntan por las metáforas que hay detrás de los usos que realizan los docentes en sus clases. Stevenson (2008) identifica cuatro metáforas al respecto: ordenadores como herramientas, como tutores, como entornos de aprendizaje y como recursos a disposición de profesores y estudiantes.

Las tres últimas metáforas son asimilables a los ejemplos que se han presentado. Los aspectos comunicativos fuera del aula son una faceta del ordenador como herramienta de comunicación (junto al uso de procesador de textos), la de repositorio y banco de recursos es equivalentes a la metáfora de recurso, y los de actividades de construcción colaborativa de conocimiento a la metáfora de entorno.

Todos los estudiantes confirmaron que los profesores utilizan las TIC en sus prácticas, pero con variaciones en esos usos.

Cerrando esa panorámica de usos, cabe indicar que los principales recursos tecnológicos utilizados y mencionados por los participantes como parte de la enseñanza son los siguientes: ordenador, internet, correo electrónico, software de presentación electrónica PowerPoint, CD-ROM, proyector multimedia, plataforma interactiva y softwares que generan mapas conceptuales.

\section{La motivación con el uso de las TIC}

Además de los tipos de usos evidenciados, que nos ha permitido situar a profesores y estudiantes y a los que remitiremos de inmediato para concretar este análisis, hemos encontrado en los datos y al reconstruir los casos unas narraciones o discursos que obedecen a una tipología similar a la triada habermasiana tan recurrente en muchos análisis de usos pero con algunas implicaciones o matices propios (Cuadro 1). De forma complementaria, aparecen también en los datos discursos esencialistas y abiertos sobre la docencia universitaria de la educación ambiental (Cuadro 2). Estas narrativas pueden ayudarnos a comprender mejor la situación de la motivación en los usos de las TIC. 
Paredes, J.; Arruda, R. D.

Cuadro 1. Discursos implicados en el análisis de la motivación propiciada por las TIC en la enseñanza.

\begin{tabular}{|l|l|}
\hline \multicolumn{1}{|c|}{$\begin{array}{c}\text { Discursos } \\
\text { subyacentes }\end{array}$} & \multicolumn{1}{c|}{$\begin{array}{c}\text { Tipología identificada sobre la percepción de las TIC } \\
\text { para la comunicación y la motivación }\end{array}$} \\
\hline $\begin{array}{l}\text { Discurso sobre } \\
\text { cualidades de los } \\
\text { dispositivos }\end{array}$ & $\begin{array}{l}\text { a) tienen características específicas o intrínsecas, tales como la misma motivación, } \\
\text { que las convierten en herramientas privilegiadas para transformar las formas de } \\
\text { relación con la información y posiblemente el conocimiento. }\end{array}$ \\
\hline $\begin{array}{l}\text { Discurso } \\
\text { simbólico-práctico }\end{array}$ & $\begin{array}{l}\text { b) pueden ser un obstáculo o un factor coadyuvante de procesos de comunicación } \\
\text { "más natural". La naturalización de la vida académica es la principal motivación. } \\
\text { Algunos van más allá, "comprometidos", con las TIC como factor de cambio. }\end{array}$ \\
\hline Discurso liberador & $\begin{array}{l}\text { c) pueden constituirse en factores de cambio, y ser la palabra una herramienta en el } \\
\text { modo de organizar el conocimiento, ayudando a construirlo. El poder de la } \\
\text { herramienta para el cambio es un poderoso acicate. }\end{array}$ \\
\hline Discurso social & $\begin{array}{l}\text { d) pueden ser formas y prácticas de organización social (ADELL, 2001), una cultura } \\
\text { comunicativa, con formas adheridas a los usos sociales de las TIC que contagian las } \\
\text { prácticas educativas cuando esas herramientas son llevadas a las aulas. La identidad } \\
\text { de las clases y lo social es la motivación básica. }\end{array}$ \\
\hline
\end{tabular}

Fuente: Elaboración propia a partir de los datos de la investigación.

Cuadro 2. Discursos esencialistas y abiertos sobre la docencia universitaria de la educación ambiental.

\begin{tabular}{|l|l|}
\hline \multicolumn{1}{|c|}{ Discursos subyacentes } & \multicolumn{1}{c|}{ Tipología identificada sobre educación ambiental } \\
\hline Discurso esencialista & $\begin{array}{l}\text { a) estar vinculada a la propia naturaleza de los estudios y al disfrute de la } \\
\text { práctica de los principios de la Educación Ambiental }\end{array}$ \\
\hline Discurso abierto & $\begin{array}{l}\text { b) responder a la incorporación de los principios de enseñanza centrados en la } \\
\text { actividad de los estudiantes por el hecho de que España ha optado por la } \\
\text { adopción del Espacio Europeo de Educación Superior para su sistema } \\
\text { educacional, con lo que las características de su enseñanza cambian y, de } \\
\text { entrada son más motivantes para los estudiantes. }\end{array}$ \\
\hline
\end{tabular}

Fuente: Elaboración propia a partir de los datos de la investigación.

Veamos con algo más de detalle estos discursos:

\section{Discurso sobre cualidades de los dispositivos}

Encontramos la idea de que los dispositivos son portadores de cualidades intrínsecas que los convierten poco menos que en mágicos. Por ejemplo, un estudiante (E1) destaca la importancia de la utilización de nuevos códigos portados por las herramientas tecnológicas, que, además de motivar, auxilian en la comprensión de los contenidos y, por consiguiente, en la construcción de conocimiento.

No hemos encontrado opiniones tan rendidas a los dispositivos entre los docentes, quizá por la madurez de los propios docentes al analizar las situaciones de aula, quizá por observar los dispositivos como meras herramientas, e importar más los procesos comunicativos, de los que se habla a continuación. 


\section{Discurso simbólico y práctico}

Más allá de lo artefactual, encontramos docentes y estudiantes provenientes o encaminados hacia el mundo de la enseñanza preocupados por los simbólico. Para ejemplificarlo se trae la opinión de tres estudiantes. En la primera, del E2, se observa que el uso de las TIC puede traer ventajas, pero demuestra una postura resistente a su uso:

Personalmente, a pesar de ver las ventajas del uso de las TIC, soy partidario de una educación más personalizada, especialmente en el ámbito de la Educación Ambiental. Asi que mi motivación es mayor cuando no se utilizan las TIC. (Extracto de la entrevista, E2)

El estudiante E2 está preocupado por los procesos comunicativos en el aula.

Una segunda opinión, favorable a un uso crítico y con criterio de las TIC, viene del estudiante E4:

Depende del propósito, mi motivación está influenciada en función de si considero que aquello para lo que va a usar (o vamos a usar las TIC) es mejor hacerlo así o de otra forma. Por ejemplo, creo que abusar de las presentaciones de PowerPoint en las estrategias docentes no me motiva a participar en la clase. (Extracto de la entrevista, E4)

Una tercera opinión, del estudiante E7, expresa una actitud favorable con relación al uso de las TIC. Preguntado sobre el nivel de motivación que siente al participar de proceso de enseñanza con uso de las tecnologías, contestó de la siguiente forma: "Uso de TIC = más motivación; clase teórica = más aburrimiento." (Extracto de la entrevista, E7).

Esas situaciones ejemplificadas, con tres posturas distintas sobre la motivación con el uso de las TIC, permiten que se tenga noción de los retos presentes en una práctica docente que se pretenda motivadora al integrar el uso de las TIC, pues la dificultad de intentar promover una mediación que contemple, en una única asignatura, distintas posturas es enorme. Sobre tal dificultad, Cabero (2006) argumenta que el profesor debe estar consciente de que el uso de esos nuevos medios requiere de la existencia de una nueva configuración del proceso didáctico y metodológico, en la cual el modelo unidireccional de formación sea sustituido por modelos más abiertos y flexibles, haciendo que los estudiantes también estén conscientes de la necesidad de ese cambio y asuman la parte del protagonismo que les otorga el proceso educativo.

Pero la renuencia de los estudiantes y profesores parece algo más profundo y menos ligado a esta exigencia comunicativa, más práctico. Algún profesor piensa que debe ejercer su papel de despertar en el estudiante la motivación cognitiva, o sea, estimularlo a pensar, y, aún más, hacerlo entender que el pensar es uno de los componentes más importantes en el aprendizaje.

La naturaleza comunicativa de las relaciones en el aula ha sido mediada por dispositivos de variada naturaleza. Estudiantes y profesores han apreciado la calidad de la comunicación y están preocupados por encontrar situaciones de enseñanza-aprendizaje más activas, por ricas y comunicativas, que potencian el crecimiento interior de los estudiantes y el aprendizaje. 


\section{Discurso liberador}

Este discurso centrado en la importancia de la comunicación está agotado para algunos profesores. Entonces quieren ir más allá; el profesor P5 dice que hace parte de su metodología llevar contenidos prácticos a los estudiantes. El aspecto que se destaca en la opinión del profesor se refiere al hecho de que la función y las características de las TIC (en este caso la naturaleza visual de los contenidos) contribuyen a la motivación del estudiante pero, más importante, a compartir el protagonismo del proceso de enseñanza entre profesor y estudiante, rompiendo con el enfoque tradicional, centrado únicamente en el profesor.

El profesor dota, además, de un uso comunicativo unas TIC cuya naturaleza inicial es presentar información, las resignifica e induce un proceso de mejora de la comunicación y las relaciones en su propia aula.

La oportunidad que tiene el estudiante de utilizar sus propias fotografías digitales en una presentación le otorga un papel importante y motivador en el proceso de enseñanzaaprendizaje, en la medida en que, con ellas, puede crear y transmitir mensajes pero, sobre todo, se otorgan nuevos papeles dentro de la comunicación en el aula, desarrollar una mayor (o un nuevo tipo de) competencia comunicativa (MARÍN, 2005). Además permite una nueva epistemología en el aula, porque los estudiantes construyen conocimiento válido en esos procesos de comunicación.

Para cerrar el análisis de la opinión del profesor P5 se hace otro destaque, que es sobre la práctica metodológica que él viene desarrollando con el uso de los recursos tecnológicos, o sea, él confirma ("está funcionando") que las TIC efectivamente contribuyen en el proceso educativo como elementos motivadores.

\section{Discurso social}

Algunos profesores y estudiantes son conscientes de que la sociedad contemporánea ha cambiado cuando ha adoptado las TIC, como plantea Castells (1999). En parecidos términos se expresa Adell (2001), destacando que además esos estudiantes llevan las TIC a las aulas y contagian con sus usos a los docentes. Algunos profesores, como el profesor P1, son conscientes de ello:

Las TIC pueden ser un elemento motivador muy importante para los estudiantes actuales porque son un elemento relevante de su cultura, basada ahora más en la imagen, la rapidez, la simultaneidad, etc. En ese sentido, son un recurso que conecta bien con sus maneras de pensar y actuar. Creo que muchos de estos elementos son comunes a la mayoría de los jóvenes [...]. De todas formas, estoy de acuerdo en que entre los estudiantes existen desigualdades que se manifiestan en sus distintos niveles de motivación y que hay que atenderlas en la formación diversificando los recursos y estrategias que se utilizan (y que se solicita utilizar). (Extracto de la entrevista, P1)

Además de resaltar el valor de algunas características de las TIC, como la utilización de imagen, la rapidez y la simultaneidad, el participante trae otro aspecto importante para la discu- 
sión, la "cultura del estudiante", de utilizar los recursos, comentando que ese uso está ya "integrado" a su modo de actuar y pensar. Esa "cultura del estudiante" puede ser entendida como un reflejo de la presencia de las tecnologías en todas las esferas de la sociedad, o sea, que el estudiante lleva al aula su práctica social cotidiana, extraescolar, donde se utilizan más las TIC.

Sin embargo, el profesor P1 resalta que, debido a las desigualdades entre los estudiantes (tanto de competencias para utilizar las herramientas, como las sociales, o aún de postura ante las TIC), la motivación se presenta en niveles distintos. Y es justamente en ese punto que el papel del profesorado, como mediador del proceso de construcción del conocimiento, se hace importante y necesario, en la medida en que, identificando tales desigualdades, deberá actuar para "atenderlas en la formación diversificando los recursos y estrategias que se utilizan (y que se solicita utilizar)" (Extracto de la entrevista, P1).

\section{Discurso esencialista}

Para algunos profesores, quizá muchos, lo importante es la materia:

El profesor P9 es categórico al afirmar que la motivación debe ser la Educación Ambiental. Indica que sería un equívoco considerar que la motivación deba ser originada con las TIC. Para argumentar ese su pensamiento, presenta varias acepciones de Educación Ambiental ("el cambiar el mundo, el discurso utópico, las formas de actuar, lo que se propone, las acciones que se establecen vivenciales") (Extracto de la entrevista, P9) que, estas sí, deben ser consideradas motivaciones.

Sin embargo, en la exposición de su opinión, presenta algunas cualidades y características de las TIC mencionando, al respecto del contacto con distintas experiencias, que sin los recursos tecnológicos no sería posible tal hecho, que sería una motivación lo que generó una duda en la comprensión de su opinión. Así lo han expresado Ojeda, Gutiérrez y Perales (2009, p. 319):

[...] tal vez porque en esta parcela educativa se considera incuestionable el contacto directo con la realidad, con el medio natural y con los problemas reales del medio ambiente que nos rodea, siendo difícil sustituirlos por la 'realidad virtual' que proporcionan las TIC.

Con el objetivo de contemplar las posturas más renuentes, se hace el siguiente cuestionamiento: si el uso de las TIC en la práctica educativa ofreciera la viabilidad necesaria para la efectiva resolución de problemas relativos a la Educación Ambiental, ¿utilizar tales recursos sería entonces motivante en la enseñanza de sus gestores y profesionales educativos? Este discurso cala entre los estudiantes en un discurso militante como el que exhibía el estudiante E2 cuando se refería a las TIC como despersonalizadoras.

\section{Discurso abierto}

Algunos profesores y estudiantes son renuentes a la introducción de las TIC en la enseñanza universitaria. En primer lugar, a la necesidad de adecuación a las "exigencias" derivadas de las propuestas del Proceso de Bolonia ${ }^{3}$. 

las TIC.

Un cambio por una imposición legal no crea las mejores perspectivas para el uso de

Sin embargo, son precisamente los postulados de una enseñanza centrada en la actividad del estudiante los que abren nuevos espacios a los usos de las TIC, en un discurso abierto en el que se establece un diálogo entre docentes, contenidos y estudiantes gracias a otras propuestas metodológicas.

Aparecen pues divergencias sobre el hecho de que las TIC se constituyan en elementos motivadores para el proceso de enseñanza-aprendizaje.

\section{Discusión y conclusiones}

En síntesis, algunos hallazgos presentados han sido que:

a) Hay poderosas narrativas en los centros universitarios que están condicionando la integración de las TIC. Ciertamente, aunque hay algunos estudiantes que no desean cambiar su estatus en una enseñanza transmisiva en la que insisten sus docentes, parece que bastantes están deseosos de un cambio de la enseñanza al ser ésta el campo profesional en el que se proyectó su formación y algunos, con buen criterio, están hastiados de prácticas tradicionales encubiertas en usos populares de TIC.

b) Una de las narrativas que destaca con fuerza es la simbólica y práctica, donde los profesores perciben que algunos cambios y resignificaciones de las TIC inducen mejoras de la comunicación educativa, lo que a su vez aumenta la motivación de sus estudiantes.

c) Las TIC pueden constituirse en factores de cambio en el modo de organizar el conocimiento, precisamente al tener esa funcionalidad y ser adoptadas por los profesores, lo que induce un deseo de transformar la enseñanza mediante la construcción de conocimiento y donde se hace muy atractivo este proceso a los estudiantes.

d) Las TIC obedecen a prácticas de organización social, con formas adheridas a los usos sociales de las TIC que contagian las prácticas educativas cuando esas herramientas son llevadas a las aulas. Sin embargo, los diferentes grados de alfabetización de los estudiantes neutralizan los efectos motivantes del uso de herramientas y códigos habituales entre ellos.

e) Hay también profesores que, identificando intuitivamente estos factores que se han puesto de relieve y deseosos de acercar las prácticas de su aula a las prácticas y lenguajes

\footnotetext{
${ }^{3}$ El denominado Proceso de Bolonia introduce diversas modificaciones en la legislación que regula el currículo (organización por competencias, duración de los Grados, transversalidad, verificación de estudios por una agencia independiente, carga de trabajo del estudiante, movilidad entre estudios nacionales y europeos, especificaciones en el título) y las actividades de enseñanza-aprendizaje (enseñanza centrada en el estudiante, tutela académica, evaluación) en la universidad española. Hay información exhaustiva en $<\mathrm{http}: / /$ www.queesbolonia.gob.es>. La renuencia se manifiesta por una supuesta mercantilización de los estudios (su vinculación al mundo empresarial por las competencias) junto al esfuerzo por pasar de una enseñanza tradicional a otra más activa, en la que los usos de las TIC tienen una oportunidad.
} 
cotidianos de sus estudiantes, o para mejorar los procesos de comunicación en el aula, promueven innovaciones acompañadas de TIC. Sin embargo, algunos docentes y estudiantes de estas titulaciones apuntan una supuesta contradicción entre discursos sobre Educación Ambiental y prácticas con tecnología.

Para terminar, y reflexionar sobre la temática de los cursos, la Educación Ambiental, hemos de decir que como tal conjunto de disciplinas, la Educación Ambiental ha quedado enfrentada a la motivación mediante TIC por aquellos docentes (actuales y futuros) que han hecho suyo un discurso esencialista. Sin embargo, aquellos que adoptan los discursos liberador y abierto encuentran una ligazón positiva entre motivación mediante TIC y Educación Ambiental, bien por encontrar coincidencias entre los fines de la propia Educación Ambiental y de las prácticas sociales y educativas con TIC, bien por la naturaleza de las propias prácticas educativas que gustan adoptar en sus clases.

Entonces, los datos apuntan a que no hay consenso entre los participantes sobre el aspecto de la motivación en los procesos formativos con el uso de las TIC. Entre el profesorado, la mayoría cree que las TIC traen motivación al proceso de enseñanza-aprendizaje, pero algunos divergen de esa opinión, diciendo que la motivación debe ser el propio contenido y finalidades de la formación, la Educación Ambiental o, incluso, el propio proceso cognitivo reflexivo que deriva del proceso formativo. Al traer la mirada de los estudiantes para esa discusión, nuevamente no hubo consenso, pues las opiniones se dividen prácticamente en partes iguales, o sea, la mitad está de acuerdo que las TIC motivan el proceso de enseñanza y la otra mitad es discordante.

Todo ello nos hace pensar que la motivación con el uso de las TIC aparecerá cuando se incluya en la enseñanza lo que Mallart (2008) ha denominado principios básicos de actuación de una enseñanza que busca la motivación de los estudiantes, unos 40, y esos principios sean coherentes con las estrategias de acción utilizadas por el profesorado: la encrucijada de estudiantes alfabetizados y que aceptan un discurso nuevo, en espacios para reconocerse, mejoras de los procesos de comunicación, implicación en tareas y proyecto de construcción de conocimiento, donde quede claro que TIC, las prácticas de enseñanza e intervención en Educación Ambiental no sean contradictorios.

Pero, tal y como ocurre en el caso analizado, a ello se llegará sólo si las TIC están integradas en la formación de esos educadores. 
Paredes, J.; Arruda, R. D.

\section{Referencias}

ADELL, J. Tendencias en educación en la sociedad de las tecnologías de la información. In: AREA, M. (Coord.). Educar en la sociedad de la información. Bilbao: Editorial Desclée de Brouwer, 2001. p. 103-198.

ALONSO-TAPIA, J. Motives, expectancies and value-interests related to learning: the MEVA questionnaire. Psicothema, Oviedo, v. 17, n. 3, p. 404-411, 2005.

ALONSO-TAPIA, J. Evaluación de la motivación en entornos educativos. In: ÁLVAREZ M.; BISQUERRA, R. (Ed.). Manual de orientación y tutoría. Barcelona: Praxis, 2007. p. $210-224$.

ALONSO-TAPIA, J.; HUERTAS, J. A.; RUIZ, M. A. On the nature of motivational orientations: implications of assessed goals and gender differences for motivational goal theory. The Spanish Journal of Psychology, Madrid, v. 13, n. 1, p. 231-242, 2010.

BARDIN, L. Análise de conteúdo. Lisboa: Edições 70, 2008.

BOEKAERTS, M.; NIEMIVIRTA, M. Self-regulated learning: finding a balance between learning goals and ego-protective goals. In: BOEKAERTS, M.; PINTRICH, P.; ZEIDNER, M. (Ed.). Handbook of self-regulation. San Diego: Academic Press, 2000. p. 417-450.

BORONAT, J. Análisis de contenido. Posibilidades de aplicación en la investigación educativa. Revista Interuniversitaria de Formación del Profesorado, Zaragoza, v. 19, n. 2, p. 157-174, 2005.

CABERO, J. Nuevas tecnologías aplicadas a la educación. Madrid: McGraw Hill, 2006.

CASTELLS, M. La era de la información. Madrid: Alianza, 1999. 3 v.

CEBREIRO, B. Las nuevas tecnologías como instrumentos didácticos. In: CABERO, J. Tecnología educativa. Madrid: McGraw Hill, 2007. p. 159-172.

COX, M.; PRESTON, C.; COX, K. What motivates teachers to use ICT? In: BRITISH EDUCATIONAL RESEARCH ASSOCIATION ANNUAL CONFERENCE, 1999, Brighton. Disponible en: <http://www.leeds.ac.uk/educol/documents/00001329.htm>. Consulta en: 18 feb. 2011.

DE PABLOS, J. Los medios como objeto de estudio preferente para la tecnología educativa. In: __ Tecnología y educación: una aproximación sociocultural. Barcelona: Cedecs, 1996. Disponible en: <http://www.lmi.ub.es/te/any96/depablos_cedecs/>. Consulta en: 07 ago. 2006.

GARCÍA PASCUAL, E. Motivación del profesorado universitario para el uso de las Tecnologías de la Información y la Comunicación (TIC) en el acto didáctico. Anuario de Pedagogía, Zaragoza, n. 4, p. 165-196, 2002.

GOETZ, J. P.; LECOMPTE, M. D. Etnografía y diseño cualitativo en investigación educativa. Madrid: Morata, 1988. 
La motivación del uso de las TIC en la formación ...

HENDRIKS, P. Why share knowledge? The influence of ICT on the motivation for knowledge sharing. Knowledge and Process Management, Chichester, v. 6, n. 2, p. 91-100, 1999.

HERNÁNDEZ, R.; FERNÁNDEZ, C.; BAPTISTA, P. Metodología de la investigación. México: McGraw-Hill, 2006.

KARSENTI, T. Comment le recours aux TIC en pédagogie universitarie peut favoriser la motivation des étudiants: le cas d'un cours médiatise sur le web. Cahiers de la Recherche en Éducacion, Québec, v. 4, n. 3, p. 455-484, 1999.

KARSENTI, T.; LIRA-GONZALES, M. El impacto de un curso en línea obligatorio en el perfil motivacional de futuros profesores. RED. Revista de Educación a Distancia, n. 22, 2010. Disponible en: <http://dialnet.unirioja.es/servlet/articulo?codigo=3267559>. Consulta en: 17 feb. 2011.

KARSENTI, T.; LIRA-GONZALES, M. La importancia de la motivación y las habilidades computacionais de los futuros profesores en el uso de las TIC. RIES: Revista

Iberoamericana de Educación Superior, Huixquilucan, v. 2, n. 3, 2011. Disponible en: $<$ http://ries.universia.net/index.php/ries/article/viewArticle/87/lira>. Consulta en: 17 feb. 2011.

LÉVY, P. Cibercultura. 2. ed. São Paulo: 34, 2000.

MARÍN, V. Comunicación mediada por tecnologías. In: CEBRIÁN, M. (Coord.).

Tecnologías de la información y comunicación para la formación de docentes. Madrid: Pirámide, 2005. p. 41-56.

MALLART, J. Didáctica de la motivación. In: DE LA HERRÁN; PAREDES (Coord.).

Didáctica general. Madrid: McGrawHill, 2008. p. 177-196.

MCCLINTOCK, R. Experience and innovation: reflections on emerging practice with new media in education. Journal of Educational Computing Research, Farmingdale, v. 25, n. 1, p. 5-104, 2001. Disponible en: <http://pocketknowledge.tc.columbia.edu/home.php/ viewfile/2407>. Consulta en: 24 nov. 2009.

MERMA MOLINA, G. Competencias del profesorado para el uso de las tecnologías de la información y la comunicación en la enseñanza, en el marco del espacio europeo de educación superior. In: ROIG VILA, R. I.; BLASCO MIRA, J. E. (Coord.). Investigación e innovación en el conocimiento educativo actual. Alcoy: Marfil, 2008. p. 317-326.

MINAYO, M. C. S. El desafío del conocimiento: investigación cualitativa en salud. Buenos Aires: Lugar Editorial, 1995.

OJEDA, F. Educación ambiental y tecnologías de la información y la comunicación: diseño, desarrollo y evaluación de un programa colaborativo en educación secundaria. Málaga: Editorial de la Universidad de Granada, 2008. 
Paredes, J.; Arruda, R. D.

OJEDA-BARCELÓ, F.; GUTIÈRREZ-PÉREZ, J.; PERALES-PALACIOS, J. ¿Qué herramientas proporcionan las tecnologías de la información y la comunicación a la educación ambiental? Revista Eureka sobre Enseñanza y Divulgación de las Ciencias, Cádiz, v. 6, n. 3, p. 318-344. Disponible en: <http://redalyc.uaemex.mx/src/inicio/ ArtPdfRed.jsp?iCve=92013010002>. Consulta en: 24 ago. 2009.

PAREDES, J.; ESTEBANELL, M. Actitudes y necesidades de formación de los profesores ante las TIC y la introducción del crédito europeo: un nuevo desafío para la educación superior. Revista de Educación, Madrid, n. 337, p. 125-148, 2005.

PUGLISI, M. L. Análise de conteúdo. 2. ed. Brasília: Liber, 2007.

STAKE, R. E. Investigación con estudio de casos. 4. ed. Madrid: Morata, 2007.

STEVENSON, I. Tool, tutor, environment or resource: exploring metaphors for digital technology and pedagogy using activity theory. Computers \& Education, London, v. 51, n. 2, p. 836-853, 2008. Disponible en: <http://portal.acm.org/ citation.cfm?id=1375283\&coll> . Consulta en: 06 ene. 2010.

TEJEDOR, F. J.; GARCÍA-VALCÁRCEL, A. Competencias de los profesores para el uso de las TIC en la enseñanza: análisis de sus conocimientos y actitudes. Revista Española de Pedagogía, Madrid, v. 64, n. 233, p. 21-43, 2006.

TEJEDOR, F. J.; GARCÍA-VALCÁRCEL, A.; PRADA, S. Medida de actitudes del profesorado universitario hacia la integración de las TIC. Comunicar, Huelva, v. 17, n. 33 , p. $115-124,2009$.

Artigo recebido em 27/12/2011. Aceito em 05/02/2012. 